DOI: $10.17234 / S R A Z .65 .16$

UDK: 811.13-05 Skok, P. UDK: 811.13:371.3

Review article

Reçu le 30 avril 2020

Accepté pour la publication le 25 novembre 2020

\title{
Petar Skok - prédécesseur de la didactique des langues étrangères contemporaine
}

\author{
Yvonne Vrhovac \\ Faculté de philosophie et lettres \\ Université de Zagreb \\ yvonne.vrhovac@zg.t-com.hr
}

Nous allons présenter l'ouvrage de Petar Skok intitulé „Méthodologie de la langue française“(édité à Zagreb en 1939) et nous soulignerons les idées principales qui concernent $l^{\prime}$ enseignement des langues étrangères. Pour Skok l'objectif principal de l'enseignement d'une langue étrangère est de rendre l'usage de cette langue efficace pour l'apprenant, c'est à dire de permettre à la personne qui apprend une langue étrangère de parler cette langue. Dans notre approche nous allons comparer les idées de Skok avec les réflexions actuelles menées en didactique contemporaine. Nous montrerons en quoi Skok peut être considérer comme précurseur de la didactique actuelle. Chaque contenu abordé par Skok est décrit en détail à l'aide d'une approche contrastive comparant ainsi les contenus de la langue étudiée avec les mêmes contenus dans la langue maternelle afin de souligner ainsi les similarités et les différences entre les deux langues.

Mots clés: enseignement des langues étrangères, objectifs, méthodes, pratique, didactique actuelle

\section{Présentation de l'ouvrage}

L'ouvrage de Petar Skok intitulé „Méthodologie de la langue française“ (Zagreb, 1939) est composé de deux parties: Partie générale et Partie spéciale. Dans la Partie générale se trouvent les chapitres „Regards sur l'enseignement des langues étrangères“, „Expériences personnelles de l'auteur"et „Littérature du sujet". La Partie spéciale contient les chapitres: „La théorie de la méthodologie de la langue française“, et "La méthodologie de la langue française en pratique" (la prononciation, l'orthographe, le vocabulaire, la morphologie, la syntaxe, de la traduction, de l'apprentissage de la littérature française et l'introduction à la culture générale). Nous allons nous concentrer plutôt sur La Partie générale, et examiner le chapitre "Regards sur l'enseignement des langues étrangères". Dans cette partie l'auteur s'exprime d'abord sur l'utilisation des deux différentes méthodes d'enseignement des langues étrangères, utilisées à cette époque - 
méthode directe ou méthode indirecte. La méthode directe utilisait des images, l'enseignant s'en servait pour poser des questions du type "Qu'est-ce que c'est?" l'apprenant devait alors répondre en fonction de l'image "C'est une table", ou „C'est une chaise" et à la question “"Où est la table?" il devait dire „, La table est à gauche", en s'exprimant directement en langue étrangère sans recourir à sa langue maternelle. D'où le nom de méthode directe. La méthode semi-directe qui avait été introduite dans les écoles à cette même époque pour l'enseignement de l'allemand et du français consistait en combinaison des deux méthodes et était selon Skok la solution à privilégier. Skok pose ensuite une question intriguante: Peut-on apprendre une langue étrangère à l'école? Il y répond en insistant sur la nécessaire prise de conscience des enseignants de leur vécu et de leurs expériences personnelles. Skok formule sa conclusion de façon un peu ironique en affirmant que la seule langue qu'il a appris à parler à l'école est le serbo-croate littéraire. Il a appris l'allemand à l'aide de la méthode traditionnelle, le français à l'aide de la méthode semi-directe, utilisée dans le manuel d'Adamović avec les images de Hölzel, il a appris l'italien seul à la Faculté de Vienne par une méthode déjà vieillie. La seule langue qu'il a bien apprise en situation didactique est le roumain appris à la Faculté de Vienne à l'aide d'un excellent professeur. Skok conclut alors que la meilleure approche pour l'apprentissage d'une langue est l'énergie personnelle, l'assiduité et la persévérence des apprenants. Voici donc pour Skok les meilleurs chemins qui mènent au succès. Il nous semble approprié de comparer ces idées de Skok avec les courants de pensées récents sur les auto-apprentissages et le rôle de l'apprenant dans la pédagogie actuelle des langues étrangères. On s'interroge pour mieux cerner les personnes qui décident d'apprendre une langue étrangère seules, qui sont-elles et pourquoi ce choix ? Comment font-elles et à l'aide de quels intermédiaires ? Ils existent aujourd'hui différents supports et outils conçus pour l'auto-appentissage des langues qui n'existaient pas aux temps de Skok. On parle aujourd'hui bien sûr de multimédias, de TIC qui mettent à la disposition de l'apprenant des contenus ou activités pédagogiques très variés pour l'auto-apprenant mais aussi des conseils et des suggestions de travail pour l'enseignant. Nous sommes tout à fait conscient que l'accès à une langue étrangère est facilité aujourd'hui par les technologies modernes à disposition et qu'il s'agit d'une pédagogie active puisque qu'elle repose sur la volonté et la motivation personnelle de l'apprenant. Celui-ci se sent responsable de ses progrès, de ses résultats et prend en charge son apprentissage. L'autonomie est donc un moyen mais en même temps un objectif. L'apprenant fait preuve de ses habilités à se débrouiller tout seul sans enseignant comme le fait tout être humain maître de ses choix. C'est ainsi que s'est comporté Skok pendant ses études à Zagreb et à Vienne. En parlant de l'autonomie il ne faut pas oublier de prendre en considération les composantes sociales et surtout psychologiques qui sont des parties incontournables de la personnalité de l'apprenant. La construction de la personnalité de l'apprenant est intimement liée à ses facultés d'autonomie et à un passage par un apprentissage sans le guidage d'un enseignant. 


\section{Méthodes de travail}

Skok expose ensuite des méthodes de travail qui mènent au succès. Chaque fois que cela est possible il faut utiliser la langue étrangère pendant le travail en classe. La grammaire même doit être enseignée en langue étrangère, il est nécessaire d'exclure complètement la langue maternelle du travail en classe. Développer le dialogue entre l'enseignant et les apprenants en langue étrangère est impératif. Il est indispensable de réfléchir au type de communication dont l'apprenant aura un jour besoin. Pensons à introduire dans le travail de classe de petits poèmes, poésies pour les apprenants débutants avec l'objectif de faire acquérir des éléments phonétiques de la langue française notamment la prononciation, l'intonation, le rythme. Il est important de travailler sur les textes du manuel à l'aide de questions et de réponses afin d'habituer les apprenants au dialogue. Pour un meilleur apprentissage il est bon d'illustrer les mots nouveaux à l'aide d'images en excluant autant que possible le recours à une explication en langue maternelle en classe. Au niveau plus avancé de l'école secondaire il est recommandé de demander aux apprenants de préparer à la maison et en langue étrangère quelques contenus de matières non linguistiques. On peut y voir les tendances actuelles se rapportant à l'interdisciplinarité - la transdisciplinarité. Il faut essayer de rassembler des contenus de disciplines différentes, la langue étrangère devient alors un instrument de transmission de ces savoirs et un véritable outil de communication. L'enseignant ne devrait jamais travailler sans un plan de travail. Il devrait planifier son travail non seulement pour une leçon donnée ou une séquence mais le construire en cohérence pour toute l'année scolaire. Ce genre de travail nécessite une forte implication, des réflexions renouvellées, cette façon d'enseigner est dure et épuisante et on suggère aux personnes fragiles et sensibles de ne pas se vouer à la profession de professeur.

Parlant des méthodes de travail Skok pose cette question importante: Quels sont les objectifs de l'enseignement d'une langue étrangère? D'abord permettre aux apprenants de bien comprendre la langue étgrangère, la première étape étant d'aiguiser leur ouie pour ensuite devenir capable de parler cette langue, en s'exerçant à s'exprimer oralement. Le travail sous forme de dialogue permet de développer ces objectifs. Il faut prendre en considération la petite taille de notre pays et sa faible population. Quand on prend l'exemple d'une personne éduquée, d'un intellectuel, on envisage que celle-ci est censée connaître au moins une langue étrangère. Ces idées de Skok nous font penser aux objectifs de la politique linguistique du Conseil de l'Europe qu'on trouve dans le Cadre européen commun de référence pour les langues :

„que c'est seulement par une meilleure connaissance des langues vivantes européennes que l'on parviendra à faciliter la communication et les échanges entre Européens de langue maternelle différente et, partant, à favoriser la mobilité, la compréhension réciproque et la coopération en Europe et à éliminer les préjugés et la discrimination" (Cadre 2000, p. 10)

Skok poursuit ses réflexions et nous donne à voir sa vision de l'importance de connaître des langues étrangères. L'apprentissage d'une langue étrangère s'appuit 
sur l'idée de rendre les jeunes personnes capables de comprendre différentes cultures contemporaines. Les langues étrangères servent à appréhender et comprendre différentes cultures et à les rapprocher à travers la littérature, l'histoire, les moeurs, les caractéristiques de la vie quotidienne d'un peuple étranger. En parlant de ce sujet Skok emploie ici le terme allemand - Kulturkunde qui est aussi utilisé aujourd'hui pour parler de culture générale et d'autres éléments culturels.Voici quelques idées actuelles sur le sujet:

- La conscience interculturelle - la connaissance, la conscience et la compréhension des relations, (ressemblances et différences distinctives) entre "le monde d'où l'on vient "et "le monde de la communauté cible" sont à l'origine d'une prise de conscience interculturelle. Elle s'enrichit également de la conscience qu'il existe un plus grand éventail de culutres que celles véhiculées par les L1 et L2 de l'apprenant.(Cadre,2000, p.83)

L'interculturel suppose l'échange entre les différentes cultures, l'articulation, les connexions, les enrichissements mutuels.... Le contact effectif des cultures différentes constitue un apport où chacun trouve un supplément à sa propre culture (Dictionnaire de didactique du français) (DDF)

\section{Formation des enseignants}

Skok s'intéresse ensuite à la formation des enseignants. Il souligne que les jeunes personnes ne sont pas suffisamment formées pour exercer la profession d'enseignant. Il estime que les cours suivis par les étudiants à la Faculté sont incomplets. Pour être bon enseignant, les étudiants devraient passer au moins un séjour plus ou moins long à l'étranger. Notre futur enseignant n'est pas assez formé d'un point de vue méthodologique ni pédagogique. Il devrait passer un examen de psychologie et de pédagogie et effectuer un stage pédagogique expérimental de deux ans. En 1916 on a introduit à la Faculté un cours intitulé „Des méthodes d'enseignement des langues modernes“, il était prévu d'y exposer et d'y débattre des problèmes psychologiques et d'offrir aux étudiants quelques suggestions pratiques concernant l'enseignement. Malheureusement, un seul candidat s'est inscrit et présenté à ce cours. Voilà ce que la didactique actuelle pense des changements dans la profession de l'enseignant.

- Le statut de l'enseignant a changé dans la mesure où sur sa formation académique vient se plaquer une formation professionnelle qui consiste essentiellement à apprendre certaines habilités formelles et techniques et l'on souhaite qu'il acquière une formation pluridisciplinaire, du moins interdisciplinaire (Galisson,1980, p. 81)

Les apprenants étaient mécontents des cours qu'ils recevaient et les enseignants également puisque eux aussi vivaient un échec dans leur travail, ainsi l'idée d'une réforme de l'enseignement est née. Voilà ce que Skok dit de la profession d'enseignant: "cette classe sociale aujourd'hui aussi bien qu'avant la guerre est la principale détentrice de notre littérature et de notre travail intellectuel. L'enseignant 
est idéaliste et en comparaison avec les enseignants des écoles secondaires des autres pays, notre enseignant occupe la pire position matérielle en Europe."

Skok essaye de trouver les raisons de cette situation difficile liée à l'enseignement des langues étrangères. La profession d'enseignant est souvent exercée par des personnes qui ne possèdent pas de motivation intrinsèque. Les classes sont bondées d'apprenants (50 apprenants). Comment alors régler la discipline, comment exercer la communication et développer la collaboration entre les élèves? Si pour enseigner on utilisait la méthode moderne une classe ne pourrait pas compter plus de 20 à 30 élèves. L'enseignant n'aurait alors pas de problèmes avec la discipline, il pourrait exercer la communication et développer la collaboration entre les élèves - en instaurant par exemple le travail de groupe. L'enseignant ne possède pas d'aides pédagogiques, il ne dispose juste que du manuel. L'enseignement des langues étrangères n'a pas répondu aux attentes du public qui a été plutôt décu. Il est entré dans le moule de l'enseignement des langues anciennes. On peut voir la fonction de la classe de langue dans la citation ci-dessous.

- La classe est un lieu de prise de parole où l'individu s'exprime en tant que personne. Les échanges se développent librement, entre pairs et de gré à gré, ils se décentralisent. (Galisson, 1980, p.52)

Mécontent de la situation de l'enseignement des langues érangères de son époque, Skok offre deux solutions possibles. La première - les auteurs des manuels devraient tenir compte des problèmes méthodologiques et composer des exercices spécifiques pour certaines unités linguistiques à travailler en classe. La deuxième - l'enseignant devrait être capable d'utiliser des idées méthodologiques théoriques pour les mettre en pratique. Il devrait posséder une routine méthodologique. Skok a publié en 1918 ses expériences d'enseignement dans un article intitulé „Mes regards sur l'enseignement des langues étrangères“ ("Moji pogledi na nastavu stranih jezika") dans la revue Nastavni vjesnik,

Quelques nouveautés ont, malgré les obstacles, pu être introduites dans l'enseignement des langues étrangères comme par exemple la mise en place de cours modèles qui ont été extrêmement utiles aux enseignants. Pour montrer sa compétence à professer, l'enseignant doit se présenter à un examen de pratique professionnelle après deux ans d'expérience d'enseignement en classe. Il doit faire un cours pour prouver sa compétence pratique et se présenter à l'examen de pédagogie. A cause d'un grand manque de manuels méthodologiques, Skok a rassemblé ses articles publiés dans Nastavni vjesnik en 1918 et Prosvetni Glasnik en 1924 dans un manuel unique en y ajoutant des unités bibliographiques pertinentes.

\section{Présentation détaillée de l'ouvrage}

Skok présente maintenant son manuel de façon plus détaillée. La Partie spéciale contient les chapitres suivants „La méthodologie de la langue française en théorie " et "La méthodologie de la langue française en pratique" (la prononciation, l'orthographe, le vocabulaire, la morphologie, la syntaxe, de la traduction, l'apprentissage de la littérature française et l'introduction à la culture générale). 
„La méthodologie de la langue française en théorie“ comporte, elle aussi deux parties :

I Principes de base de la méthodologie:

a) l'objectif qu'on aimerait atteindre par l'enseignement,

b) le but général de l'enseignement secondaire

II Matière de la langue française

I Parmi les principes essentiels de l' enseignement il faut souligner

a) L'enseignement actuel des langues étrangères a un objectif pratique - il faut apprendre à parler. Pour atteindre cet objecif primordial il faut prendre en considération plusieurs facteurs - le nombre d'heures de cours par semaine, la méthode de travail utilisée, l'énergie de l'enseignant, l'intérêt que l' enseignant éveille auprès de ses apprenants, l'ambition et l'assiduité de l'apprenant. L'objectif idéal de l'enseignement du français serait qu'après huit ans d'apprentissage de la langue française l'apprenant soit capable d'exprimer oralement et par écrit et de façon grammaticalement correcte ses idées et ses connaissances acquises dans différentes domaines.

Pour l'organisation de l'école secondaire Skok propose trois niveaux: le niveau élémentaire de la première à la troisième classe ( 1 - 3 classe), le niveau intermédiaire de la quatrième à la sixième classe (4 - 6 classe) et le niveau avancé avec la septième et huitième classe (7 - 8 classe). Quelque soit le niveau l'apprenant suivra 3 heures de langue étrangère par semaine. Skok décrit également en détail le contenu de ce que l'apprenant devrait acquérir par niveau - de la langue de tous les jours jusqu'à la lecture des oeuvres littéraires.

b) L'enseignement secondaire à pour objectif général d'affermir la volonté et de former l'intellect de l'apprenant, en développant son goût pour la culture moderne. Pour atteindre ces objectifs l'enseignant devrait développer auprès de l'apprenant son sens de l'observation et ses facultés à tirer des conclusions.

La méthodologie à mettre en place pour enseigner la langue française doit découler de réflexions sur les moyens d'atteindre de façon la plus aisée possible les objectifs d'utilisation pratique de la langue apprise, comment répondre facilement aux exigeances de l'enseignement secondaire, en prenant en considération la structure du français (car on n'enseigne pas toutes les langues étrangères de la même façon). Voici quelques considérations actuelles sur le travail en classe:

- "Les didacticiens d'aujourd'hui cherchent à tenir deux buts de la chaîne, c'est-à-dire bien connaître le "à qui ?" pour mieux maîtriser le "comment ?" Ce qui les amène à une prise en compte, aussi fine que possible de tous les facteurs qui conditionnent l'acte éducatif" (Galisson, 1980, p.46)

II Matière de la langue française

Pour bien accomplir la tâche de l'enseignement secondaire il faut réfléchir à des facteurs suivants: 1 prononciation, 2 mots, 3 formes, 4 syntaxe.

Puisque du point de vue pratique la langue sert à parler, à communiquer quotidiennement il faut tenir compte des facteurs ci-dessus mentionnés. Dans l'enseignement du français dans notre pays des erreurs ont été commises, le plus souvent au niveau de la prononciation et du vocabulaire. L'apprenant 
prononçait les voyelles françaises en imitant les 5 voyelles de la langue serbocroate. Quant au vocabulaire il était enseigné à la manière des langues anciennes. L'apprenant cherche le mot inconnu dans le dictionnaire, l'écrit dans son cahier et l'enseignant demande à l'apprenant de lui dire le mot. Cette façon monotone de travailler le vocabulaire provoque l'ennui aussi bien auprès des apprenants que des enseignants. La méthode d'apprentissage des mots nouveaux devrait avoir une fonction mentale en procédant par de techniques de mémorisation dynamique, par l'évocation des mots en contexte réel et l'association d'idées. En sus de la prononciation et du vocabulaire il faut maitriser les règles de syntaxe. La syntaxe fait appel à de nombreux éléments pédagogiques. L'apprentissage de la grammaire par coeur n'a pas de composantes pédagogiques, de par son aspect répétitif, il ne permet pas à l'apprenant de mener de véritables réflexions. C'est la raison pour laquelle il faut enseigner la grammaire à l'aide d'une approche inductive basée sur l'observation suivie de son interprétation et de conclusion.

Voici les réflexions sur l'enseignement/apprentissage de la grammaire des didacticiens actuels:

- "Pour enseigner la grammaire il faut avoir "une approche assez souple qui tient compte à la fois de l'intérêt de faire découvrir par soi-même le fonctionnement de la langue et de pouvoir en parler, du fait que chaque apprenant a ses habitudes de travail et de réflexion.." (Tagliante, 1994, p.151)

- Nous avons appelé exercices de conceptualisation une démarche qui s'appuie sur la perception métalinguistique que les apprenants peuvent avoir de la languecible. Il s'agit moins d'exercices que de moments de réflexion grammaticale explicitée par les apprenants" (Besse, 1984, p.113)

Skok décrit aussi comment enseigner la littérature au secondaire et comment transmettre la culture générale aux apprenants. Au niveau le plus élevé l'apprenant devrait acquérir des connaissances sur les époques les plus importantes en littérature et sur leur histoire. Ainsi l'apprenant confronté aux textes littéraires pourra les analyser à l'aide de ses observations et de son vécu de façon inductive et non pas à l'aide d'idées ou de phrases dictées.

Acquérir des connaissances sur la culture française et les cultures des pays étrangers (Skok utilise aussi le terme allemad Auslandskunde) se trouve déjà au programme de la 4e classe. Les textes abordent la culture française, Paris, l'histoire et la vie quotidienne. Mais les textes sont trop compliqués et trop difficiles. Le contenu des textes abordés traite de thèmes comme par exemple Les Français ont de l'esprit, ils sont nationalistes, l'administration est centralisée, et on trouve le régionalisme seulement en dernier. Skok trouve que ces sujets sont trop compliqués pour les apprenants de 4 e classe et en plus le curriculum ne mentionne pas quand et comment travailler sur ces thèmes.

\section{Conclusion}

Notre intention a été qu'en exposant les idées principales de l'ouvrage Méthodologie de la langue française de Petar Skok publié à Zagreb en 1939 de mettre 
en avant comment ce grand linguiste des langues romanes a été préoccupé par les idées générales de l'enseignement des langues étrangères dans le système scolaire de l'époque. Il s'est soucié du contenu de l'enseignement et surtout de la la façon dont on enseignait les langues étrangères - des méthodes de travail employées en son temps. En comparant certaines des pensées de Skok avec les idées et les approches actuelles en didactique des langues étrangères, nous espérons que nous avons réussi à montrer que dans certains domaines avec ses idées révolutionnaires Skok n'a pas été seulement partisan du progrès mais surtout un précurseur des tendances et des approches récentes en didactique.

\section{Bibliographie:}

Barbot, Marie-José (2000). Les auto-apprentissages, Paris: CLE International Besse, Henri., Porquier, Rémi (1984). Grammaires et didactique des langues, Paris: LAL, Hatier-Crédif

Cadre de référence pour les langues (2000). Conseil de l'Europe, Strasbourg

Cuq, Jean-Pierre (2003). Dictionnaire de didactique du français langue étrangère et seconde, Paris: CLE International (DDF)

Galisson, Robert (1980). D'hier à aujourd'hui la didactique générale des langues étrangères, Paris: CLE International

Helmling, Brigitte (2002). L'apprentissage autonome des langues en tandem, Paris:Didier

Little, David (2002). Autonomie de l'apprenant, Paris: Didier

Tagliante, Christine (1994). La classse de langue, Paris: CLE International

\section{Petar Skok - prethodnik suvremene glotodidaktike}

Analizom Skokova djela „Metodologija francuskoga jezika“ (Zagreb, 1939.) iznijet ćemo osnovne Skokove ideje koje se odnose na nastavu živih jezika, npr. živi se jezik obućava s praktičnim ciljem kako bi se naučilo govoriti. Cilj je nastave da se nakon 8 godina školovanja učenik zna gramatički korektno izraziti na pismenoj i usmenoj razini. Osim toga, cilj je nastave da se pomoću jezika obrazuje učenikov intelekt i volja. Stoga nastavnik francuskoga jezika ne smije razmišljati samo o praktičnim ciljevima nastave već mora kod učenika razviti smisao za opažanje i zaključivanje. Ove Skokove misli usporedili smo s principima suvremene metodike nastave stranih jezika. Nakon osnovnih misli Skok određuje materiju koju valja metodički obraditi, a to su izgovor, riječi, oblici i sintaksa. Svakom ovom području Skok posvećuje iscrpan opis služeći se često kontrastivnim pristupom uspoređujući ove sadržaje s istim sadržajima u materinskom jeziku naglašavajući sličnosti i razlike. Na kraju svakog odjeljka nalazi se iscrpan popis relevantnih bibliografskih jedinica. Zadnji odlomak u knjizi bavi se pitanjima učenja francuske književnosti i upoznavanja francuske kulture općenito.

Ključne riječi: nastava stranih jezika, ciljevi , metode, suvremeni pristup nastavi stranih jezika 